Utah State University

DigitalCommons@USU

\title{
A Pilot Randomized Controlled Trial of Online-Delivered ACT- Enhanced Behavior Therapy for Trichotillomania in Adolescents
}

\author{
Michael P. Twohig \\ Utah State University, michael.twohig@usu.edu \\ Julie M. Petersen \\ Utah State University, julie.petersen@aggiemail.usu.edu \\ Jeremiah Fruge \\ Utah State University, jeremiah.fruge@usu.edu \\ Clarissa W. Ong \\ Utah State University, clarissa.ong@usu.edu \\ Jennifer L. Barney \\ Utah State University, jen.barney4@aggiemail.usu.edu \\ Jennifer Krafft \\ Utah State University, jennifer.krafft@aggiemail.usu.edu \\ Follow this and additional works at: https://digitalcommons.usu.edu/psych_stures \\ eprext dqa月e for achditional authorisology Commons
}

\section{Recommended Citation}

Twohig, M. P., Petersen, J. M., Fruge, J., Ong, C. W., Barney, J. L., Krafft, J., Lee, E. B., \& Levin, M. E. (2021). A Pilot Randomized Controlled Trial of Online-Delivered ACT-Enhanced Behavior Therapy for Trichotillomania in Adolescents. Cognitive and Behavioral Practice.

This Article is brought to you for free and open access by the Psychology Student Works at DigitalCommons@USU. It has been accepted for inclusion in Psychology Student Research by an authorized administrator of DigitalCommons@USU. For more information, please contact digitalcommons@usu.edu.

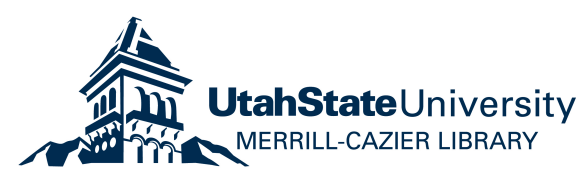


Authors

Michael P. Twohig, Julie M. Petersen, Jeremiah Fruge, Clarissa W. Ong, Jennifer L. Barney, Jennifer Krafft, Eric B. Lee, and Michael E. Levin 
A Pilot Randomized Controlled Trial of Online-Delivered ACT-Enhanced Behavior Therapy for Trichotillomania in Adolescents

\author{
Michael P. Twohig, Ph.D. ${ }^{\text {a }}$ \\ Julie M. Petersen ${ }^{\text {a }}$ \\ Jeremiah Fruge $^{\text {a }}$ \\ Clarissa W. Ong ${ }^{\text {a }}$ \\ Jennifer L. Barney ${ }^{\mathrm{a}}$ \\ Jennifer Krafft ${ }^{\text {a }}$ \\ Eric B. Lee, Ph.D. ${ }^{b}$ \\ Michael E. Levin, Ph.D. ${ }^{\text {a }}$ \\ ${ }^{\text {a }}$ Utah State University \\ ${ }^{\mathrm{b}}$ Southern Illinois University
}

\title{
Author note:
}

This study was supported by funding from the Huntsman Foundation. Please address correspondence to Michael Twohig at Michael.twohig@usu.edu. Handouts and other materials are available at UtahACT.com. 


\begin{abstract}
Treatment of adolescent trichotillomania is understudied, and treatment providers are difficult to find. In this pilot study, we compared ACT-enhanced behavior therapy to a waitlist, with treatment delivered over Zoom, to 28 adolescents who met criteria for trichotillomania. This study partially occurred during the COVID-19 pandemic. A detailed description of the treatment protocol is provided for ACT-enhanced behavior therapy adapted to a Zoom format for adolescents. Pretreatment assessments were completed over Zoom by an assessor unaware of study condition; pretreatment and posttreatment questionnaires were completed online. Results indicated that therapy was delivered with fidelity and competence over Zoom, with $100 \%$ session completion. Adolescents reported that pulling severity decreased significantly more in the treatment condition than waitlist. No differences were seen in other adolescent or parent-reported pulling severity or distress. However, significant medium to large within-condition effect sizes were observed in the treatment condition. Medium effect size changes were seen in the treatment group on a measure of trichotillomania-related psychological flexibility, and a small effect size was seen for psychological distress. Overall, pilot results provide preliminary support for ACTenhanced behavior therapy, delivered over Zoom, for adolescent trichotillomania, indicating the need for a fully powered confirmatory efficacy trial.
\end{abstract}


A Pilot Randomized Controlled Trial of Online-Delivered ACT-Enhanced Behavior Therapy for Trichotillomania in Adolescents

Trichotillomania involves the excessive pulling out of one's hair to the point of noticeable hair loss and unsuccessful attempts to control the pulling, along with resulting distress and/or impairment in important life domains (APA, 2013). Even though hair can be pulled from any part of the body, the most common pulling area is the scalp (APA, 2013; Franklin et al., 2008). Trichotillomania has a 1-3.5\% prevalence for adolescents (Christenson, Pyle, \& Mitchell, 1991; Franklin, Edson, \& Freeman, 2010) and is associated with depression, anxiety, and poor social and academic functioning (Franklin et al., 2008).

There are useful interventions for adolescents with trichotillomania. Thus far, the most promising intervention is Habit Reversal Training (Franklin, Edson, Ledley, \& Cahill, 2011; Rahman, McGuire, Storch, \& Lewin, 2017). Habit reversal training involves helping the client become aware of the pulling and the actions that precede pulling, engaging in an alternative when pulling occurs or is about to occur (called a "competing response"), and implementing a reinforcement program to maintain the competing response. Eight sessions of habit reversal training were compared to approximately three hours of non-therapeutic therapist contact $(\mathrm{N}=24)$, and researchers found the active condition resulted in significantly greater reduction in pulling (75\% responders) over the control condition (0\% responders, Franklin et al., 2011). A similar treatment was compared to treatment as usual in 40 adolescents, where it was again found that habit reversal resulted in greater decreases in pulling (76\% responders) over the treatment as usual group (21\% responders, Rahman et al., 2017).

One of the methods investigated as an adjunctive to habit reversal training is acceptance and commitment therapy (ACT); this combination is also called ACT-enhanced behavior therapy 
(Woods \& Twohig, 2008). The logic behind this intervention is that the ACT procedures would assist with motivation to address pulling and provide skills to respond more effectively to urges or cognitions that traditionally facilitate pulling. ACT teaches psychological flexibility - the ability to notice inner experiences for what they are, allow them to exist, and continue moving in meaningful life directions. Psychological inflexibility has established positive associations with trichotillomania (Begotka, Woods, \& Wetterneck, 2004; Houghton et al., 2014), even mediating the relationship between affect and severity of pulling (Houghton et al., 2014). Furthermore, because models of trichotillomania support multiple types of pulling, focused (conscious) and unfocused (unconscious), it is theorized that ACT-enhanced behavior therapy better addresses both types of pulling (Woods \& Twohig, 2008). Therefore, ACT-enhanced behavior therapy may be especially fitting because most individuals show elements of both types of pulling, even if they engage in one more often than the other (Flessner et al., 2007).

The initial study of ACT-enhanced behavior therapy for trichotillomania was a multiple baseline study with six adults. Pulling reduced to near zero levels for four of the six participants, with results maintained for three participants at three month follow-up (Twohig \& Woods, 2004). This study was followed by a randomized controlled trial with 25 adults; $66 \%$ of those in the treatment condition were responders as compared to $8 \%$ of the waitlist (Woods, Wetterneck, \& Flessner, 2006). The same manual (Woods \& Twohig, 2008) was also translated to Finnish and tested in a group format with 53 adults (Haaland et al., 2017). Strong results were found with $90 \%$ of participants responding at posttreatment and $60 \%$ of participants maintaining gains at one-year follow-up. More recently, ACT-enhanced behavior therapy was tested as an online intervention for adults $(\mathrm{N}=22)$, delivered over Zoom with corresponding measures and handouts delivered over the internet (Lee, Haeger, Levin, Ong, \& Twohig, 2018). Results were very 
similar to in-person studies even though the intervention was not completed in-person. More specifically, the treatment group had a $42 \%$ decrease in pulling (58\% responders) and the waitlist had a $17.7 \%$ increase in pulling ( $0 \%$ responders). This study raises important considerations because delivering treatment online circumvents some key issues that are faced in the treatment of trichotillomania.

In general, online treatment or teletherapy sidesteps many of the issues that exist in faceto-face therapy including access to providers, social stigma involved in attending therapy, and need to commute to therapy sessions (Maheu, Pulier, McMenamin, \& Posen, 2012). Teletherapy has also become the standard in response to the COVID-19 pandemic, where face-to-face sessions greatly increase the risk of transmission of the virus, endangering therapist, client, and possibly the community. Teletherapy is also pertinent to the treatment of trichotillomania due to the incredible shortage of therapists trained in the treatment of this disorder (Woods et al., 2006). Even though teletherapy may only solve the therapist shortage within the state where the therapist is licensed, it still offers access to services more so than in-person services, particularly for rural or otherwise low-access areas.

As discussed earlier, there is some research on the treatment of adolescents with trichotillomania, but the scope is more limited than the work with adults. More specifically, although we have evidence that habit reversal training is useful for adolescents with trichotillomania, many adolescents do not respond (Franklin et al., 2011; Rahman et al., 2017). To date, only one case series tested ACT-enhanced behavior therapy in this age group; both participants demonstrated clinically significant gains with periods of no pulling over two weeks (Fine et al., 2012). Additionally, one randomized trial tested ACT alone in the treatment of trichotillomania in a combined sample of adolescents and adults (Lee et al., 2020). The adults 
reduced their pulling by $58 \%$ whereas adolescents reduced pulling by $31 \%$. Similarly, there were larger decreases in psychological inflexibility in the adults $(36 \%)$ than the adolescents $(11 \%)$. These results suggest that ACT alone can be helpful for adolescents; however, ACT plus habit reversal training may be a more suitable treatment for adolescents because stronger results were seen with the case series (Fine et al., 2012). ACT-enhanced behavior therapy has never been tested in a randomized trial for adolescents.

We chose to test this intervention delivered over an online platform because the difficulties in accessing treatment is even greater for adolescents than adults with trichotillomania (Franklin et al., 2008). Furthermore, the results from Lee and colleagues' (2018) trial provided confidence that this treatment could be successfully delivered online. We made age appropriate modifications to the protocol (originally designed for adults) and its delivery method. For example, we taught skills with new metaphors and exercises to match their developmental level and emphasized habit reversal training, contingency management, and token economy to match the understanding that psychological inflexibility may be less of an issue at lower developmental levels. Additionally, the COVID-19 pandemic overlapped with the end of our enrollment period and we were able to continue the study uninterrupted, thus highlighting one of the additional benefits of telehealth as a delivery method.

This study tests ACT-enhanced behavior therapy delivered over teletherapy in 28 adolescents diagnosed with trichotillomania in a pilot randomized controlled trial. Assessors who were unaware of treatment condition conducted a diagnostic interview over Zoom and measures of trichotillomania severity (main outcome measure), psychological distress (to assess for related important behavior change), and psychological flexibility (predicted process of change in ACT for trichotillomania) were also collected online. Therapy was conducted over 10 sessions through 
Zoom; caregivers were provided handouts for each session and participated in the final minutes of each session. We predicted that ACT-enhanced behavior therapy would result in reductions in trichotillomania and problem behavior as measured by standardized measure and an increase in psychological flexibility over the waitlist control. We also predicted that delivering treatment over Zoom would be feasible, result in high scores of treatment integrity, and demonstrate acceptability as measured by low drop-out.

\section{Method}

\section{Participants}

The sample consisted of 28 adolescents and at least one of their caregivers. Adolescent participants were predominantly female $(68 \%)$ with ages ranging from 12 to $16(\mathrm{M}=13.9, \mathrm{SD}=$ 1.3). Within the sample, $16(57.1 \%)$ participants met criteria for at least 1 comorbid diagnosis. The most common comorbid diagnoses were depressive episodes $(42.8 \%)$ and anxiety disorders (ranging from $7-10 \%$ of the sample; see Table 1 for more detail). Many (40\%) of the sample was on a SSRI and $26 \%$ were taking stimulant medication for ADHD.

Participants were recruited from online postings, advertisements in newspapers, and referrals from other professionals. Eligibility and exclusion criteria included the following: (a) met DSM-5 criteria for trichotillomania; (b) seeking treatment primarily for trichotillomaniarelated concerns; (c) was 12 to 17 years old; (d) was fluent in English; (e) was not currently receiving psychotherapy; (f); had not started or changed psychotropic medication in the past 30 days; (g) was not planning to start or change psychotropic medication during the course of the study; (h) had not completed high school; and (i) residing in the state where the lead author is licensed. Two participants met initial screening but when intake materials were completed, they reported no pulling and were not enrolled. A third participant completed intake but then ran away 
from home and was unreachable, so the parent withdrew her from the study. See Figure 1 for a CONSORT diagram of participant flow into and through the study.

\section{Power Analysis}

A power analysis was conducted using G*Power software (Faul, Erdfelder, Lang, \& Buchner, 2007) in order to determine the number of participants to include in the study. A past trial of ACT-enhanced behavior therapy for trichotillomania resulted in a large effect size $(d=$ .81; Woods et al., 2006). Therefore, a power analysis was performed using this same effect size, with alpha set at .05 and power at .80 , in a repeated measure analysis, specifying a sample of 24 . A slightly more conservative effect size estimate of .70 resulted in a sample size of 28.

\section{Study Procedure}

The study was approved by a university internal review board. Participants were randomized into either a treatment or waitlist control group. Randomization occurred through randomizing numbers 1-30 and assigning odds and evens to each group. The condition was only revealed to the assessor after the participant completed intake and was deemed eligible for the study. Thus, pretreatment semi-structured interviews were completed by an assessor who was unaware of treatment condition. All participants eventually received treatment, with those in the waitlist group waiting 12 weeks before beginning treatment. Participants and caregivers completed full assessment batteries at pre-treatment/pre-waitlist and at post-treatment/postwaitlist. All assessments and treatment sessions took place online using the Qualtrics survey platform and Zoom video conferencing software.

Therapists were four graduate students in a clinical psychology program and their supervisor (XXX, who is an author of the treatment manual and a licensed psychologist). Each 
person served as the therapist for approximately 5 participants (participants who completed waitlist also received treatment). Weekly supervision (by XXX), including video review, occurred.

\section{Caregiver Involvement}

Caregivers were invited to participate in the final 10 minutes of each session. This allowed for the adolescent to review what they had learned and also allow the caregiver to be involved in the fulfillment of treatment goals. Caregiver utilization of these final 10 minutes varied based on participant age, with caregivers of older children utilizing it less. There was variability in who the caregiver was (mother, father, alternating, both, client going between caregiver homes) for session feedback. Caregivers were also given a short ( $1 \frac{2}{2}$ to 1 page) handout following each session that explained what was taught and how they could best help their child to make progress. Handouts and the therapy protocol are available at removed for masked review.

\section{Intervention}

The protocol consisted of ten, 50-minute sessions that combined traditional habit reversal training and ACT, adapted from existing protocols (Lee et al., 2018; Woods \& Twohig, 2008). Generally, habit reversal techniques were used to facilitate awareness of pulling behavior and to teach skills that reduce the likelihood of pulling or make pulling more effortful to engage in. On their own, habit reversal techniques can eliminate pulling behavior; however, they tend to evoke difficult internal thoughts, emotions, and urges that can make sustained use challenging. ACT was used to help with these difficult internal experiences by fostering psychological flexibility and increasing the ability to identify and make space for urges to pull while replacing pulling behavior with valued actions. Caregivers were emailed brief, single-paged handouts before each session that provided psychoeducation about trichotillomania and parental accommodation, 
reviewed material presented in the session (e.g., ACT concepts), and gave tips to help their child succeed in completing homework and mastering skills.

Session 1: Psychoeducation and functional analysis. The first session began with a discussion of the use of technology to facilitate treatment. This discussion included the limits to confidentiality, the caregivers' ability to access information, and instructions to turn off attention-competing devices and programs (e.g., cell phones, console video games, and other programs on the computer). This portion required less time once COVID-19 quarantine occurred because students and caregivers became more familiar with Zoom. Participants were then oriented to the intervention and expectations over the course of treatment. The majority of the session was spent conceptualizing and obtaining a detailed functional assessment of the adolescent's pulling behavior. This assessment included gathering a history of emotions, urges, and thoughts that might accompany the pulling behavior, contexts in which pulling occurs, past treatments or other attempts to stop pulling, comorbid mental health conditions that might influence the pulling behavior, and family accommodation that might reinforce pulling. For example, some adolescents were unaware of the internal experiences that played into their pulling such as itching on the scalp, feelings of pressure, or worries that certain hairs would bother them until pulled out. Throughout the treatment, global life functioning and well-being were emphasized as the treatment targets, consistent with an ACT approach. The effect of COVID-19 on pulling was also explored when appropriate - adolescents reported varying differences in pulling in response to COVID-19. Family dynamics were an important consideration and were also explored in the first session. Many caregivers closely monitored pulling and caregivers often expressed frustration with the child's pulling. Pulling after a long period of resistance was often experienced as failure, leading to the caregivers and adolescents 
feeling increasingly stuck. Overall, caregivers were instructed to be more of a "supporting player" on their child's team and less of a "coach." At the end of the session, adolescents were given a form to self-monitor their daily urges and pulling behavior. Engaging the caregiver and child over Zoom can be a little more complicated than in-person. Being clear about who the therapist is speaking to helped with receiving useful responses.

Session 2: Habit reversal training and stimulus control. Habit reversal and stimulus control procedures were the focus of session 2. The awareness training portion of habit reversal aimed to help adolescents become more aware of their pulling behavior and associated antecedents that predict when pulling is likely to occur. We called these "warning signs" or "warning signals" and practiced watching for them in session. This was often framed as a game, which was an adaptation for the adolescent population. The adolescent and therapist would engage in a conversation on a topic of the adolescent's choice (e.g., sports, pets, music) and the adolescent would try to catch the therapist engaging in warning signs or pulling behavior as fast as they could. The adolescent would then try to catch their own warning signs as they occurred during the session, having them pointed out by the therapist when necessary. Next, competing response training was introduced as another key component of habit reversal. This technique simply involves engaging in a physical response, such as making fists with hands, whenever a warning sign is noticed or whenever actual pulling behavior occurs. This strategy was introduced as a way to break the habit of pulling by introducing a behavior incompatible with pulling (e.g., you can't pull your hair when your hands are in fists), not as a method for removing urges to pull. Competing responses were practiced multiple times in session. Adolescents would engage in an antecedent pulling behavior, such as playing with their hair, and then immediately practice their competing response for one minute. When the time had elapsed, if the urge to pull was still 
present, the competing response would be repeated for another minute. Adolescents were informed that practicing their competing response would likely lead to increased internal experiences related to pulling, at least in the short term, and that future sessions would cover strategies for dealing with them. Finally, stimulus control was introduced. Simply put, this procedure aims to make pulling behaviors more effortful to engage in by altering stimuli and contexts associated with pulling. For example, wearing gloves in contexts where pulling is highly likely (e.g., in the car or when watching a movie), requiring tweezers to be checked out from caregivers, or wearing hair in a ponytail when lying in bed. Adolescents were encouraged to brainstorm ideas with their caregivers and work together as a team to implement these strategies and assess their utility. Caregivers were taught to reinforce the use of competing responses and stimulus control strategies with attention and/or activities that the adolescent enjoys (e.g., extra screen time). Caregivers tended to want to tie large rewards with large, longterm goals (e.g., a new phone for a month of zero pulling) and were reoriented to principles of effective reinforcement strategies (i.e., small, immediate, and consistent). All and all, this session is similar in-person or on telehealth.

Session 3: Values exploration. The goals of session 3 included defining values, exploring the adolescent's personal values, and discussing ways in which pulling was getting in the way of valued action. Most adolescents had not previously considered the ways in which their pulling had impacted their life beyond parental dissatisfaction and social embarrassment. Values probing questions such as, "what are you excited about in your life," "when do you feel really alive," and "what would you like to do when you get older" were used to explore what was important to them. A metaphor, such as the "passengers on the bus" was used to illustrate the ways that pulling could take their focus off their values. Adolescents were asked to imagine 
driving a bus to a destination that was really important to them (their value). But then different passengers (internal thoughts, emotions, urges) get on the bus and demand attention. Often the adolescent finds themselves pulled over on the side of the road arguing and fighting (hairpulling, trying to suppress urges) with these passengers rather than actually driving to where they want to go. There is a useful cartoon video of this metaphor on the internet that can be watched together with the therapist via screensharing in Zoom. Homework was assigned to consider and write down the ways in which pulling had gotten in the way of valued action.

One unique tweak that occurs when working on values with adolescents is that clarity on what is "valued" is developmentally related to age. For example, some of the younger and less developmentally sophisticated participants could only note values such as "fun" and "friends." However, older participants would connect with values such as occupation and romantic relationships, both of which are more affected by pulling. For the younger participants, we needed to focus more on using token economy to maintain behavior change, whereas the older clients were more motivated by external social reinforcers (e.g., greater ease in dating).

Session 4: Control as the problem. Now that the adolescent had some experience using behavioral techniques for controlling their pulling (i.e., competing responses and stimulus control), attention was turned inward to the urges, emotions, and thoughts that surround the pulling. The goal of the session was to help the adolescent learn that the urge to pull and the accompanying inner experiences were not the real problem; rather, the problem came from the reaction to these experiences and from the efforts to remove or control them. It was not the passengers on the bus that was causing them problems, it was the fact that they had pulled over their bus to argue and fight with them. The adolescents were asked to identify and describe in detail what they experienced when they had an urge to pull. With adolescents, it was often 
helpful to practice in session or ask them to recount a recent experience of using their competing response. Then they were asked a question such as, "what changes, or what do you hope will change, when you pull?" Additionally, the lasting effect of the adolescent's strategies were examined. Sometimes they were able to make themselves feel better in the short term by pulling, but the urges always returned and usually only got worse over time. Again, for adolescents, this concept made sense to practice in session because they often did not notice it in day-to-day life. The adolescent was told that they would explore an alternative to controlling the urge in the next session. To set this up, the adolescent was asked to engage in a simple exercise. They were instructed to fold up a very small piece of paper, place it in their shoe, and leave it there until the next session.

Session 5: Acceptance. The paper in the shoe exercise was discussed at the start of session 5 as a metaphor for acceptance. Using their experience with the paper in the shoe, adolescents were taught that making space for the discomfort they experienced on their foot led to them eventually forgetting it was even there. Conversely, times when they wiggled their foot in their shoe and tried to control the discomfort only led to the discomfort and annoyance increasing and lasting longer. The word willingness was generally used in place of acceptance to emphasize the active role of making space for inner experiences rather than simply tolerating them. Willingness was presented as an alternative strategy to the less workable control strategy. Exercises such as having the adolescent play with their hair while trying their hardest not to have an urge to pull further illustrated the lack of control that humans have over their internal experiences. Again, teaching these skills with practice exercises seems to really help when working with adolescents over Zoom. These exercises were often surprising for the participants because they typically reacted to the urge to pull automatically. Building up the urge and simply 
sitting with it was an eye-opening experience for most participants. The passengers on the bus metaphor was expanded upon and continued to be used as a way to encourage participants to place their focus on their values, while practicing willingness to make space for their urges without pulling over to engage with them. The passengers on the bus metaphor was meaningful for many participants because many rode the bus to and from school and they were aware of bossy and pushy riders. We also used examples of "annoying" classmates and how one could still be nice to them even though they were annoying - just like one can allow urges to be there even though they can be annoying. Having the handouts and the final minutes with the caregivers was helpful because parents had previously tried to help their children control urges and this time allowed the therapist to teach them to reinforce and model willingness.

\section{Sessions 6 and 7: Defusion, present moment awareness, and continued acceptance.}

These sessions continued to reinforce willingness skills while introducing concepts of defusion and present moment awareness. Adolescents were introduced to the idea that our minds often play tricks on us using language that grabs our attention. It was explained that the passengers on the bus often use words or phrases that really push our buttons, are especially hard to make space for, and really make us to want to pull the bus over to the side of the road and fight. Given that we were presenting therapy over Zoom, we often used short video clips that showed these concepts and metaphors (via Zoom screen share). Given the popularity of ACT, there are hundreds of cartoon-like videos illustrating these points. We often used the "passengers on the bus" and "unwelcome party guest" videos. Showing those videos was a nice break from therapy for the participant, and it seemed that a quick video caught the participants attention in a new way. After the video we discussed its meaning and how it applied to their work with hair puling. Other exercises included imagining their thoughts on a movie screen while sitting in the audience 
and watching, differentiating between descriptions of the world and the evaluations that our minds make about them, and listing reasons to pull their hair while not actually engaging in the behavior. The more comfortable a therapist is with ACT, the more easily the therapist can match or adapt examples to the adolescent's interests. For example, a loud crowd at a basketball game or watching skiers from a chairlift.

Session 8 and 9: Exposure. Exposure to urges to pull were used to help participants practice willingness, defusion, and valued-action skills. They were framed as a way to "make friends with your urges" by intentionally inviting an urge without making attempts to distract from it or control it by pulling. Depending on their skill level, adolescents were instructed to invite urge sensations by imagining tingling, pressure, or other urge experiences, imagining pulling and the accompanying sensations, touching their hair and finding individual strands that they wanted to pull, and/or looking in a mirror or webcam (a benefit of Zoom) to find hair that looked out of place or wrong. This was a difficult task for many of the participants but allowed for in vivo experience of urges while having support from the therapist. For example, we had one participant who would develop small bulges on his scalp that he related to a pimple (likely and infected hair follicle). He would pull all the hairs in the surrounding area and reduce the pressure of that pimple. We worked to find a pimple and really explore what it felt like, what he was hoping for if he pulled around it, and what he worried about by not pulling. He said things like, “this pimple will bother me all day," and "I won't be able to sleep with this on my head." This exercise provided wonderful opportunities to practice defusion and acceptance exercises around those urges. We could also practice values in that moment by saying, "what might you gain by ignoring this pimple for the next week?" Homework was assigned to continue exposure practice between sessions. 
Session 10: Review and relapse prevention. The final session included relapse prevention and a review of all prior material with emphasis placed on topics that the therapist felt most applicable to the participant. Relapse prevention included a plan for continued practice of skills and noting the difference between a lapse and complete relapse. Participants were reminded that they would likely have times when they pulled again in the future and were encouraged to frame these as only brief lapses that necessitated a review of their skills and continued practice. Potential warning signs of slipping into old habits were identified with plans to take action to prevent relapse. When possible, adolescents scheduled regular check-ins with a parent to track progress and receive ongoing reinforcement. Interestingly, it is difficult for those diagnosed with trichotillomania to achieve complete symptom remission. Thus, we had a handful of participants who were still pulling at low rates at the end of treatment. We encouraged the participant and parent to continue to use the ACT, habit reversal, and contingency management skills on their journey to stopping pulling fully. We emphasized that therapy was a "big" step in that direction but not the full journey. Again, having the caregiver be part of this session was particularly helpful because adolescents are often not as good at setting and adhering to longterm plans. This session was therefore a good time to talk to the caregivers about which systems were in place.

\section{Measures}

The Mini International Neuropsychiatric Interview was given at pre-treatment. All other measures were given to adolescents and caregivers at pre- and post-treatment using an online survey platform.

\section{Mini International Neuropsychiatric Interview for Children and Adolescents 7.0.2}

(MINI-KID). The MINI-KID is a structured diagnostic interview developed for use with children 
and adolescents. It was used to assess for comorbid diagnoses at pre-treatment. The MINI-KID has demonstrated excellent psychometric properties including good sensitivity and specificity and inter-rater and test-retest reliability (Sheehan et al., 2010). The version used in this study was updated by the developers to match DSM-5 criteria.

\section{Trichotillomania Scale for Children - Child and Parent Versions (TSC-C \& TSC-P;}

Tolin et al., 2008). The TSC-C and TSC-P are 12-item, self-report measures of youth- and parent-rated trichotillomania symptom severity. Parent-child agreement between the two measures is generally good. For each item, the rater chooses the best fitting response of multiple response options. Responses are rated from 0 to 2 and are summed into two subscales: severity and distress/impairment. The TSC-C and TSC-P demonstrated good internal consistency in the current sample ( $\alpha \mathrm{s}=.89$ and .89 , respectively).

Acceptance and Action Questionnaire for Trichotillomania (AAQ-TTM; Houghton et al., 2014). The AAQ-TTM is a 9-item, self-report measure of psychological flexibility based on the Acceptance and Action Questionnaire-II (AAQ-II; Bond et al., 2011) and specifically developed for people with trichotillomania symptoms. Examples of questions include, "I am able to not pull when the urge to pull is strong," and "I am not afraid of my urges to pull." Contextspecific versions of the AAQ-II tend to perform better than the general-version (Ong, Lee, Levin, $\&$ Twohig, 2019). Items are rated on a seven-point Likert scale $(1=$ never true to $7=$ always true) that are summed into a total score (range $=7-63$ ) with higher scores indicating higher levels of psychological flexibility or lower levels of psychological inflexibility (note this is opposite of the AAQ-II). The AAQ-TTM demonstrated excellent internal consistency in the current sample $(\alpha=.91)$. 
Youth Outcome Questionnaire 30.2 Self-Report and Parent-Report Versions (YOQSR \& YOQ-PR; Burlingame, Wells, Lambert, \& Reisinger, 1998). The YOQ-SR and YOQ-PR are 30-item measures of youth- and parent-rated psychosocial distress and well-being. Items are rated on a five-point, Likert scale $(0=$ never or almost never to $4=$ always or almost always $)$ that are summed into a total score (range $=0-120$ ). Clinically significant scores for the YOQ-SR and YOQ-PR are 30+ and 29+, respectively. The YOQ-SR and YOQ-PR demonstrated excellent internal consistency in the current sample ( $\alpha \mathrm{s}=.95$ and .95 , respectively).

\section{Treatment Integrity Coding}

Treatment sessions were recorded using the built-in recording function in Zoom. We selected $20 \%$ (30 of the 150) completed sessions for treatment integrity scoring. For even distribution across sessions and participants, we ensured that at least five videos were selected per session number and two videos were selected per participant. Within these constraints, videos were selected by matching randomly selected participants to two randomly selected sessions (1 to 10, repeated five times) using a random number generator without replacement. Selected sessions were assessed for treatment integrity using a standardized scoring measure that has been used in a previous clinical trial testing ACT-enhanced behavior therapy for trichotillomania (Lee et al., 2018). Habit reversal (i.e., awareness training, competing response training, and contingency management), ACT (i.e., acceptance, defusion, present-moment awareness, self-as-context, values, and committed action), and ACT-inconsistent (i.e., cognitive challenging, experience avoidance strategies, and thoughts and feelings cause behaviors) elements were each scored on five-point Likert scales for quality and quantity from 1 (the variable was never explicitly covered) to 5 (the variable occurred with high frequency and was covered in a very in-depth manner). Global processes like adherence to the treatment model and 
therapist competency were also scored from 1 (not at all adherent/competent) to 5 (extremely adherent/competent). Two trained graduate research assistants who had used this integrity measure on previous projects and established interrater reliability with the other trained raters scored the sessions with an ICC greater than .9 (Lee et al., 2018).

\section{Statistical Analyses}

Analyses were conducted using R version 3.6.3 (R Core Team, 2020) in RStudio (RStudio Team, 2020) using the following packages: tidyverse (Wickham, 2017), lme4 (Bates, Maechler, Bolker, \& Walker, 2015), texreg (Leifeld, 2013), effects (Fox \& Weisberg, 2019), cowplot (Wilke, 2018), effsize (Torchiano, 2017), psych (Revelle, 2018), and furniture (Barrett \& Brignone, 2017).

Multilevel or mixed-effect models were used to evaluate between-group differences over time in an intent-to-treat sample on four outcomes of interest: trichotillomania severity (TSC-Y Severity and TSC-P Severity), distress due to trichotillomania (TSC-Y Distress and TSC-P Distress), mental health functioning (YOQ-Y and YOQ-P), and psychological flexibility related to trichotillomania (youth-reported AAQ-TTM). The first three outcomes had both youth-report and parent-report versions, which were tested in separate multilevel models.

Youth- and parent-reported TSC scores were our primary outcomes of interest; we considered the other three outcomes secondary but still relevant to overall well-being. Our analytic plan was preregistered at https://osf.io/dgrzy. Contrary to our preregistration, we did not analyze data from the Avoidance and Fusion Questionnaire for Youth and Parental Acceptance Questionnaire, which were also administered in this study, as they were less relevant to wellbeing. In addition, we used multilevel models instead of ANCOVAs to more effectively account for missing data (i.e., intent-to-treat analysis instead of listwise deletion). 
For each outcome variable (seven total), a series of nested models were fitted starting with a null model (only random intercepts). Then, we added only a fixed effect of time (measured in weeks as a continuous predictor) in the second model and only a fixed effect of condition (treatment versus waitlist) in the third model. The fourth model included both time and condition as independent fixed effects, and the fifth and final model specified a time $\times$ condition interaction term. All models included random intercepts for participants.

Each more complex model was compared to the previous most parsimonious model that improved model fit (e.g., if there was no difference between the null model and condition-only model, the subsequent more complex model was compared to the null model) using likelihood ratio tests to determine the best-fitting model at $p<.05$. However, given the small sample size and exploratory nature of the current study, we also reported models that fit better at $p<.10$. Final models were estimated using the maximum likelihood criterion. Unstandardized coefficients from the best-fitting models are reported; $p$-values are based on the Satterthwaite approximation to degrees of freedom.

We also calculated Hedges' $g$ effect sizes for between-group differences at posttreatment and within-group change in the treatment group from pre- to posttreatment. Generally, 0.2 corresponds to a small effect, 0.5 to a medium effect, and 0.8 to a large effect.

With regard to the participant who ran away and was withdrawn from the study, we analyzed the data with and without that participant and the results were not notably different. We chose to remove this participant from analyses to be conservative because she was in the waitlist condition and data collection following her withdrawal was not possible.

\section{Results}

\section{Treatment Integrity and Feasibility}


Means and standard deviations for treatment integrity coding of ACT processes were calculated over the course of treatment rather than by session. This is due to the built-in flexible nature of ACT wherein processes are targeted based on client presentation and an evolving case conceptualization rather than a prescribed sequence. Means for the habit reversal training processes were: awareness training $(M=2.53, S D=1.28$, range $=1$ to 5$)$, competing response training $(M=2.60, S D=1.33$, range $=1$ to 5$)$, and contingency management $(M=2.27, S D=$ 1.05 , range $=1$ to 4$)$. ACT processes were rated as follows: acceptance $(M=2.80, S D=1.37$, range $=1$ to 5$)$, defusion $(M=3, S D=1.49$, range $=1$ to 5$)$, present-moment awareness $(M=$ $1.47, S D=0.82$, range $=1$ to 4$)$, self-as-context $(M=1.20, S D=0.66$, range $=1$ to 4$)$, values $(M$ $=2.47, S D=1.25$, range $=1$ to 5$)$, and committed action $(M=2.37, S D=1.13$, range $=1$ to 5$)$. These ratings indicate all treatment processes were covered at least moderately in-depth in at least one session. On average, therapists were rated as very to extremely adherent to the ACT model $(M=4.63, S D=0.61$, range $=3$ to 5$)$ and very to extremely competent $(M=4.63, S D=$ 0.61 , range $=3$ to 5$)$.

ACT-inconsistent processes were rated as follows: cognitive challenging $(M=1.00, S D=$ 0 , all scores were 1$)$, experiential avoidance strategies $(M=1.07, S D=0.25$, range $=1$ to 2$)$, and thoughts and feelings cause behaviors $(M=1.03, S D=0.18$, range $=1$ to 2$)$, indicating minimal use of ACT-inconsistent speech.

Procedures matched the flow of the treatment manual with Sessions 1 and 2 receiving the highest mean scores for the habit reversal training processes of awareness training, competing response training, and stimulus control procedures $(\mathrm{Ms}=3.86,3.00$, and 2.71 respectively). Session 3, which was supposed to be on values, had the highest scores for the HRT processes and values (Ms ranged from 3.00 to 3.67). The key process targeted by the rest of the ACT 
sessions were in line with the manual: Sessions 4 and 5 had the highest scores for acceptance (M $=4.00)$ and Sessions 6 and 7 had the highest scores for defusion $(M=4.71)$. Sessions 8 to 10 were for exposures and relapse prevention, so processes targeted were variable and depended on the client's presentation.

All participants assigned to the treatment condition completed all ten therapy sessions, with no therapy dropouts or participants receiving fewer than ten sessions. Thus, a high degree of therapy engagement was found using the ACT-enhanced behavior therapy protocol through Zoom with adolescents.

\section{Effect of Therapy from Pre- to Post-treatment}

Regression coefficients and 95\% confidence intervals from best-fitting models are reported in Table 2. Means, standard deviations, and within-group and between-group effect sizes are reported in Table 3. A significant time by condition interaction effect was found for the primary outcome of youth-reported TSC Severity. There was a greater decrease in youth-rated TSC Severity scores in the treatment group than the waitlist group over time (see Figure 2) and a large group difference at posttreatment (Hedges' $g=1.55)$.

Significant time by condition effects were not found for other primary outcomes of trichotillomania symptoms including parent-reported TSC Severity, youth-reported TSC Distress, or parent-reported TSC Distress. Within the treatment group, there were large decreases in trichotillomania symptoms from pre- to posttreatment on these symptom measures (Hedges' $g$ $=0.85-.94)$.

Time by condition effects were also not found for the secondary outcomes of youthreported and parent-reported mental health functioning (YOQ). In the treatment group, small preto posttreatment improvements were found for mental health functioning (Hedge's $g=.39$ ). A 
non-significant trend $(p=.075)$ was found for a time by condition effect on psychological flexibility related to trichotillomania (AAQ-TTM), suggesting that scores in the treatment group increased more over time than in the waitlist group (see Figure 2). There was a medium increase in scores from pre- to posttreatment in the treatment group (Hedges' $g=-0.53$ ) indicating greater psychological flexibility.

\section{Discussion}

In this study, we tested the preliminary feasibility and efficacy of online-delivered ACTenhanced behavior therapy for adolescents diagnosed with trichotillomania in a pilot randomized controlled trial using a waitlist control condition. All participants completed $100 \%$ of therapy sessions and integrity coding demonstrated that treatment was delivered with fidelity. We found significant improvements on the primary outcome of youth-reported trichotillomania severity in the treatment group over the waitlist. Even though group differences were not seen in trichotillomania-related distress, medium to large within effect sizes were seen for the treatment group. Small between-group effects were seen for youth and adult reported mental health functioning. Finally, a non-significant between-group trend was seen on the AAQ for trichotillomania at the $p=.075$ level; the treatment group had a medium within-group effect size in the direction of greater psychological flexibility. These pilot results provide preliminary support for ACT-enhanced behavior therapy delivered online for adolescent trichotillomania and suggest the need for a larger efficacy trial.

This study is only the third randomized trial for adolescents with trichotillomania; all trials have been published since 2010, suggesting that adolescent trichotillomania is highly understudied. Both other studies also found significant decreases in the treatment condition over unmatched control conditions and all studies had non-responders and individuals who were still 
symptomatic (Franklin et al., 2011; Rahman et al., 2017). The findings from this study suggest ACT-enhanced habit reversal may be a useful alternative way to present habit reversal training. Additionally, this, study coupled with previous studies using ACT-enhanced behavior therapy for adolescents (Fine et al., 2012) and adults (Lee et al., 2018; Woods et al., 2006), suggests this line of research is worthy of further investigation.

The outcomes from this study require a deeper analysis. First, significant between-group differences were found for youth-reported trichotillomania severity. Whereas, parent-report for both these measures showed notable decreases but they were more modest than youth-report. This discrepancy should be investigated in future studies. One solution is to also include more objective measures of pulling. This might be especially pertinent with adolescents over adults. A similar interesting finding was that youth and caregivers reported lower distress scores at pretreatment than pulling severity scores. This finding might suggest that the adolescents were less distressed by their trichotillomania at pretreatment and were more pleased with gains that were not $100 \%$. This theory fits with what we observed as the therapists: in many cases, the caregivers were more concerned about the ramifications of the pulling than the adolescent was. For instance, there were many cases where the caregivers sought out treatment for their child, and we had to work to build some motivation for the adolescent's participation. Trichotillomania has ego-syntonic features to it, but for adults, the social ramifications outweigh the pleasure of pulling. However, this exchange appears to be less true for adolescents and should be incorporated into treatment.

We did not see between group differences in our measure of trichotillomania-based psychological inflexibility at posttreatment. This is consistent with Lee and colleagues' (2018) study testing ACT-enhanced behavior therapy for adults with trichotillomania. With this finding 
in mind, there are two areas that should be addressed in future research. Our measure of trichotillomania-based psychological inflexibility was normed on adults and not adolescents. Thus, it is possible that this measure is not appropriate for this younger age group. An additional concern is that psychological inflexibility may not affect pulling in an "across the board" fashion in the same way we see in adults. Again, our anecdotal experience was that psychological inflexibility was a key area of discussion for certain participants, whereas, habit reversal was really key for other participants. With adults, we have generally differentiated the emphasis on ACT and habit reversal based on whether the pulling was more focused or non-focused. With adolescents, cognitive maturity or lack of impulsiveness seemed to be related to whether ACT concepts were useful. Fine-grained research into the variables that indicate the need for cognitive versus behavioral procedures in adolescent trichotillomania is needed.

At a clinical level, this study is important because it is the first randomized trial of ACTbased behavior therapy for adolescents. It is the third ever randomized trial of the treatment of adolescents with trichotillomania. Finally, it is the second trial of teletherapy for trichotillomania and the first with adolescents. Thus, this study demonstrates that ACT-based behavior therapy can work for adolescents, giving therapists another option beyond traditional habit reversal for its treatment. It also shows that this treatment can be delivered over teletherapy which is particularly important because there is a dearth of trichotillomania providers in the USA and in-person therapy can be difficult in certain contexts (e.g., living in a rural setting or a global pandemic like COVID-19). This study concluded in May of 2020 during the COVID-19 pandemic. Thus, the final five participants were seen while public schools had moved to online instruction. We observed no interruption in services and clients were able to continue with therapy uninterrupted. As we write this paper in the midst of the COVID-19 pandemic, it has even become clearer that 
teletherapy has additional benefits in that the therapists and clients can remain isolated. The isolation prevents interruption in services when the client or therapist is in quarantine but heathy enough to engage in a therapy session. Quarantine periods can be lengthy, and teletherapy allows services to continue.

While we did not provide measures of treatment acceptability, we did not experience drop-out during treatment and participants completed all sessions. Anecdotally, we did not have notable issues with canceled appointments or no-shows. Similarly, we found high ratings for both fidelity and competence delivering the therapy protocol online for this population. These high ratings suggest that delivery of psychotherapy for adolescent trichotillomania over teletherapy is feasible. This finding is important because of the dearth of providers competent in the treatment of trichotillomania in the USA. Trained providers could offer services across the state or states in which they are licensed. We also observed that the adolescents appeared quite comfortable with the teletherapy option. Many adolescents conducted the sessions from their bedrooms or while lounging on the couch, which was particularly helpful given that these sites were often where pulling occurred.

Nonetheless, we encountered a handful of distractions that we do not experience with inperson sessions. In particular, many times clients would concurrently be on their phones, playing video games, or using the computer for things other than the therapy session. We had a few sessions where the client entered the session as a passenger in a car or otherwise not in a preferred place for a therapy session. We highly suggest setting ground rules for what actions and locations are acceptable during teletherapy. Caregivers should also be aware of these rules and assist in ensuring the client adheres to them. Still, many clients were highly engaged and took the sessions very seriously. 
Another anecdotal finding that could spur future research is that a handful of clients had a difficult time engaging in therapy, especially the ACT aspect of the therapy. While we did not do a comprehensive assessment of attention deficit hyperactivity disorder (ADHD) other than the MINI-KID, we observed ADHD-consistent behaviors or had caregivers report that the adolescent had received an ADHD diagnosis previously. Furthermore, $25 \%$ of the sample were on stimulant medications. We observed that these adolescents had a harder time describing what was occurring for them when they pulled or even really being aware of their pulling. We found we focused more on habit reversal training and contingency management with these clients. This issue did not seem related to age; it appeared more associated to levels of observed impulsivity. Thus, future work should investigate the moderating role of ADHD in the treatment of trichotillomania. It has already been shown that ADHD is the second most common comorbid diagnosis with trichotillomania after anxiety disorders (Walther et al., 2014).

As with most studies, this one offers some useful information, has some limitations, and raises some new questions. The first limitation has to do with our assessment procedures. Objective measures were not collected such as photographs of pulling sites or hair collection. We only have self-report data, although we had data from both the adolescent and the caregiver. We suggest using multiple methods of data collection in the future.

Next, we included participants from 12 to 17 years of age and adolescence involves notable differences in development. While age is not perfectly related to developmental level, developmental level likely moderated our outcomes. Specifically, those who were more mature likely benefitted more from services. This statement should be taken cautiously because motivation to control pulling was tied into all this in some way, but not always related to age. We had 12-year-olds who were mature and motivated to stop pulling, and 17-year-olds we struggled 
to get to stop playing video games and work with us. Enrolling twice as many participants and using broader, developmentally-sensitive measurements would have allowed us to determine who was likely to respond to this treatment. Relatedly, a more heterogeneous sample would assist in generalization of findings. Our sample was largely white and members of the Church of Jesus Christ of Latter Day Saints.

As seen in Table 1, we had a greater percent of participants in the treatment condition who met criteria for a comorbid diagnosis on the MINI-KID than in the waitlist condition. As this study was a pilot trial, we chose not to add possible comorbid diagnosis as a covariate. We think this finding should tell future researchers and clinicians to be aware of the likely comorbid struggles that these adolescents may encounter. If a larger efficacy trial is ever conducted, we suggest more formal assessments of ADHD and other comorbid diagnoses, along with stratifying the sample across conditions.

With regard to sample size, we engaged in intensive and expensive recruiting to achieve an $\mathrm{N}$ of 28. Trichotillomania is not rare per se, but many who struggle with it do not know to look for services. Furthermore, as noted earlier, some adolescents are not motivated to receive services for their pulling. Also, our restricted age range and requirement they reside in the same state as study therapists limited our recruitment pool. While we saw significant reductions on trichotillomania severity, many of the related parent-reports or other youth measures had notable within-condition effect sizes. Funding for a larger trial would be ideal. Relatedly, the withincondition effect size might be bolstered with booster sessions after the treatment trial. Another option might be access to other self-help tools such as websites or mobile apps to maintain engagement after therapy. 
Finally, this study only included pretreatment to posttreatment measures. We are currently collecting follow-up data out to one year and the waitlist group will finish receiving treatment and have their data collected. We are choosing to report these data now due to the meaningful findings and the need to get information out on the treatment of trichotillomania and the use of teletherapy. Future papers will report on weekly data collected during treatment and the pretreatment to posttreatment results for all 28 participants.

In summary, this is one of a thee trials where adolescents with trichotillomania are treated with psychotherapy. It is the first randomized trial of ACT-enhanced behavior therapy for adolescents. The study is notable because it was entirely administered delivered over Zoom and partially occurred during the 2020 COVID-19 pandemic. Despite these factors, we had high treatment acceptability, no treatment drop-out, and 100\% completion of therapy sessions, indicating good acceptability and feasibility. Outcomes were significant for participant reported pulling, and medium to large within-condition effect sizes were seen for parent reported pulling, psychological distress, and trichotillomania specific psychological inflexibility. A larger treatment trial of ACT-enhanced behavior therapy is needed. 


\section{References}

American Psychiatric Association. (2013). Diagnostic and statistical manual of mental disorders: DSM-5 ${ }^{\mathrm{TM}}$ (5th ed.). Arlington, VA, US: American Psychiatric Publishing, Inc.

Barrett, T. S., \& Brignone, E. (2017). Furniture for quantitative scientists. $R$ Journal, 9, 142-148.

Bates, D., Maechler, M., Bolker, B., \& Walker, S. (2015). Fitting linear mixed-effects models using lme4. Journal of Statistical Software, 67(1), 1-48. doi:10.18637/jss.v067.i01

Begotka, A. M., Woods, D. W., \& Wetterneck, C. T. (2004). The relationship between experiential avoidance and the severity of trichotillomania in a nonreferred sample. Journal of Behavior Therapy and Experimental Psychiatry, 35(1), 17-24.

Bond, F. W., Hayes, S. C., Baer, R. A., Carpenter, K. M., Guenole, N., Orcutt, H. K., ... Zettle, R. D. (2011). Preliminary psychometric properties of the Acceptance and Action Questionnaire-II: A revised measure of psychological inflexibility and experiential avoidance. Behavior Therapy, 42(4), 676-688. doi:10.1016/j.beth.2011.03.007

Burlingame, G., Wells, M., Lambert, M., \& Reisinger, C. (1998). Youth Outcome Questionnaire (Y-OQ®-30.2). American Professional Credentialing Services.

Christenson, G. A., Pyle, R. L., \& Mitchell, J. E. (1991). Estimated lifetime prevalence of trichotillomania in college students. Journal of Clinical Psychiatry, 52(10), 415-417.

Fine, K. M., Walther, M. R., Joseph, J. M., Robinson, J., Ricketts, E. J., Bowe, W. E., \& Woods, D. W. (2012). Acceptance-enhanced behavior therapy for trichotillomania in adolescents. Cognitive and Behavioral Practice, 19(3), 463-471.

Flessner, C. A., Woods, D. W., Franklin, M. E., Keuthen, N. J., Piacentini, J., Cashin, S. E., \& Moore, P. S. (2007). The Milwaukee Inventory for Styles of Trichotillomania-Child 
Version (MIST-C) initial development and psychometric properties. Behavior Modification, 31(6), 896-918.

Fox, J., \& Weisberg, S. (2019). An $\{R\}$ companion to applied regression (3rd ed.). Thousand Oaks, CA: Sage.

Franklin, M. E., Edson, A. L., \& Freeman, J. B. (2010). Behavior therapy for pediatric trichotillomania: Exploring the effects of age on treatment outcome. Child and Adolescent Psychiatry and Mental Health, 4(1), 18.

Franklin, M. E., Edson, A. L., Ledley, D. A., \& Cahill, S. P. (2011). Behavior therapy for pediatric trichotillomania: A randomized controlled trial. Journal of the American Academy of Child \& Adolescent Psychiatry, 50(8), 763-771.

Franklin, M. E., Flessner, C. A., Woods, D. W., Keuthen, N. J., Piacentini, J. C., Moore, P., . . . Wilson, M. A. (2008). The child and adolescent trichotillomania impact project: Descriptive psychopathology, comorbidity, functional impairment, and treatment utilization. Journal of Developmental and Behavioral Pediatrics, 29(6), 493-500.

Haaland, Å. T., Eskeland, S. O., Moen, E. M., Vogel, P. A., Haseth, S., Mellingen, K., .. . Hummelen, B. (2017). ACT-enhanced behavior therapy in group format for Trichotillomania: An effectiveness study. Journal of Obsessive-Compulsive and Related Disorders, 12, 109-116. doi:https://doi.org/10.1016/j.jocrd.2017.01.005

Houghton, D. C., Compton, S. N., Twohig, M. P., Saunders, S. M., Franklin, M. E., NealBarnett, A. M., ... Woods, D. W. (2014). Measuring the role of psychological inflexibility in trichotillomania. Psychiatry Research, 220(1-2), 356-361. doi:10.1016/j.psychres.2014.08.003 
Lee, E. B., Haeger, J. A., Levin, M. E., Ong, C. W., \& Twohig, M. P. (2018). Telepsychotherapy for trichotillomania: A randomized controlled trial of ACT-enhanced behavior therapy. Journal of Obsessive-Compulsive and Related Disorders, 18, 106-115.

Lee, E. B., Homan, K. J., Morrison, K. L., Ong, C. W., Levin, M. E., \& Twohig, M. P. (2020). Acceptance and commitment therapy for trichotillomania: A randomized controlled trial of adults and adolescents. Behavior Modification, 44(1), 70-91.

Leifeld, P. (2013). texreg: Conversion of statistical model output in R to LaTeX and HTML tables. Journal of Statistical Software, 55(8), 1-24. doi:10.18637/jss.v055.i08

Maheu, M. M., Pulier, M. L., McMenamin, J. P., \& Posen, L. (2012). Future of telepsychology, telehealth, and various technologies in psychological research and practice. Professional Psychology: Research and Practice, 43(6), 613.

Ong, C. W., Lee, E. B., Levin, M. E., \& Twohig, M. P. (2019). A review of AAQ variants and other context-specific measures of psychological flexibility. Journal of Contextual Behavioral Science, 12, 329-346.

R Core Team. (2020). R: A language and environment for statistical computing. Vienna, Austria: R Foundation for Statistical Computing.

Rahman, O., McGuire, J., Storch, E. A., \& Lewin, A. B. (2017). Preliminary randomized controlled trial of habit reversal training for treatment of hair pulling in youth. Journal of child and adolescent psychopharmacology, 27(2), 132-139.

Revelle, W. (2018). psych: Procedures for psychological, psychometric, and personality research. R package version 1.8.10.

RStudio Team (2020). RStudio: Integrated Development for R. RStudio, PBC, Boston, MA. http://www.rstudio.com/. 
Sheehan, D. V., Sheehan, K. H., Shytle, R. D., Janavs, J., Bannon, Y., Rogers, J. E., . . . Wilkinson, B. (2010). Reliability and validity of the mini international neuropsychiatric interview for children and adolescents (MINI-KID). The Journal of Clinical Psychiatry. 71(3), 313-326.

Tolin, D. F., Diefenbach, G. J., Flessner, C. A., Franklin, M. E., Keuthen, N. J., Moore, P. S., . . . Board, T. L. C. S. A. (2008). The Trichotillomania Scale for Children: Development and validation. Child Psychiatry and Human Development, 39(3), 331-349. doi:10.1007/s10578-007-0092-3

Torchiano, M. (2017). effsize: Efficient effect size computation. R package version 0.7.1. Retrieved from https://CRAN.R-project.org/package=effsize

Twohig, M. P., \& Woods, D. W. (2004). A preliminary investigation of acceptance and commitment therapy and habit reversal as a treatment for trichotillomania. Behavior Therapy, 35(4), 803-820.

Walther, M. R., Snorrason, I., Flessner, C. A., Franklin, M. E., Burkel, R., \& Woods, D. W. (2014). The trichotillomania impact project in young children (TIP-YC): Clinical characteristics, comorbidity, functional impairment and treatment utilization. Child Psychiatry \& Human Development, 45(1), 24-31.

Wickham, H. (2017). tidyverse: Easily install and load 'Tidyverse' packages. R package version 1.1.1. Retrieved from http://CRAN.R-project.org/package=tidyverse

Wilke, C. O. (2018). cowplot: Streamlined Plot Theme and Plot Annotations for 'ggplot2'. Retrieved from https://CRAN.R-project.org/package=cowplot.

Woods, D. W., Flessner, C. A., Franklin, M. E., Keuthen, N. J., Goodwin, R. D., Stein, D. J., \& Walther, M. R. (2006). The Trichotillomania Impact Project (TIP): Exploring 
phenomenology, functional impairment, and treatment utilization. Journal of Clinical Psychiatry, 67(12), 1877.

Woods, D. W., \& Twohig, M. P. (2008). Trichotillomania: An ACT-enhanced behavior therapy approach therapist guide. Oxford University Press.

Woods, D. W., Wetterneck, C. T., \& Flessner, C. A. (2006). A controlled evaluation of acceptance and commitment therapy plus habit reversal for trichotillomania. Behaviour Research and Therapy, 44(5), 639-656. 
Figure 1. Consort diagram of participant flow.

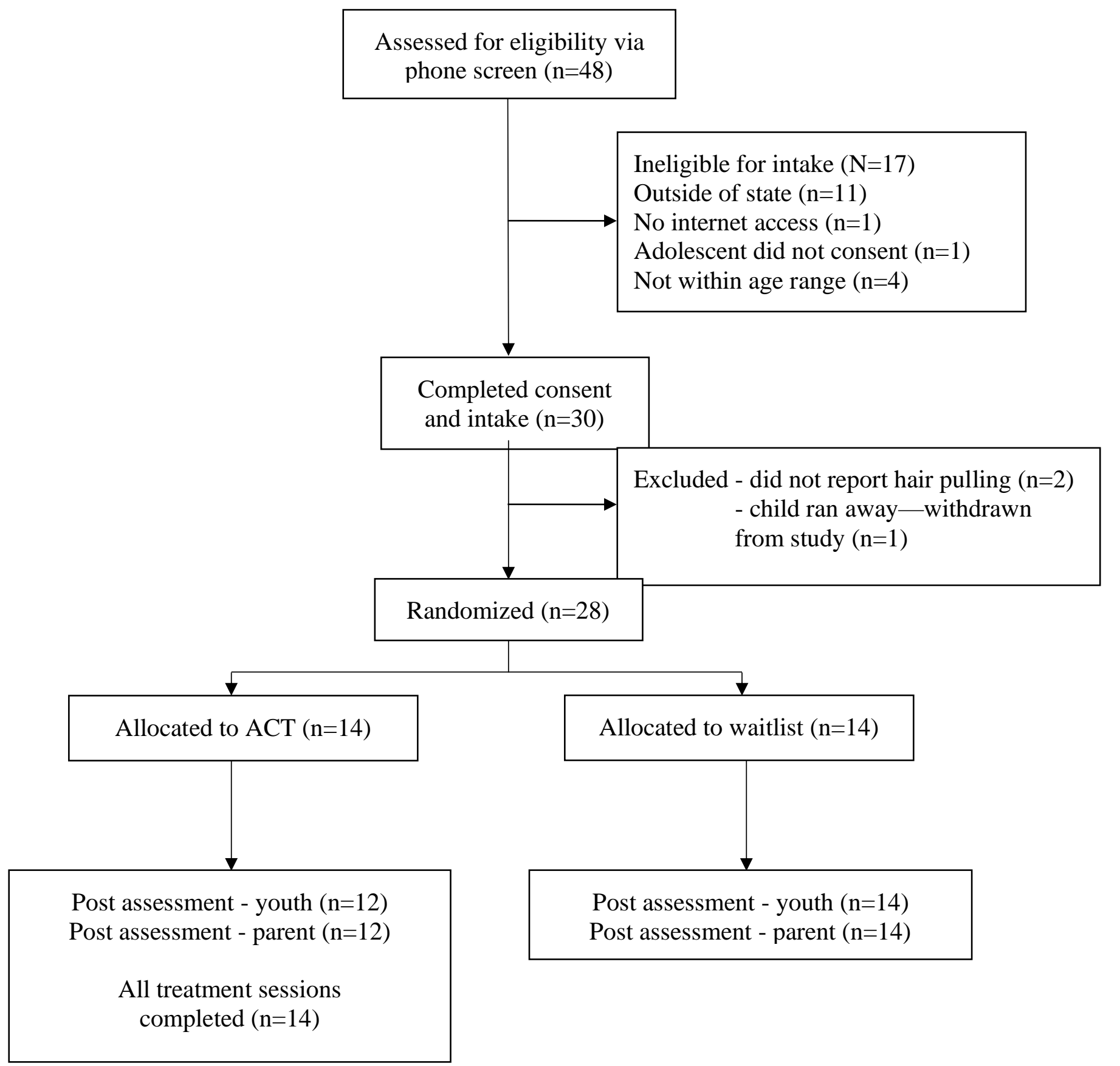


Table 1

Sample Descriptives

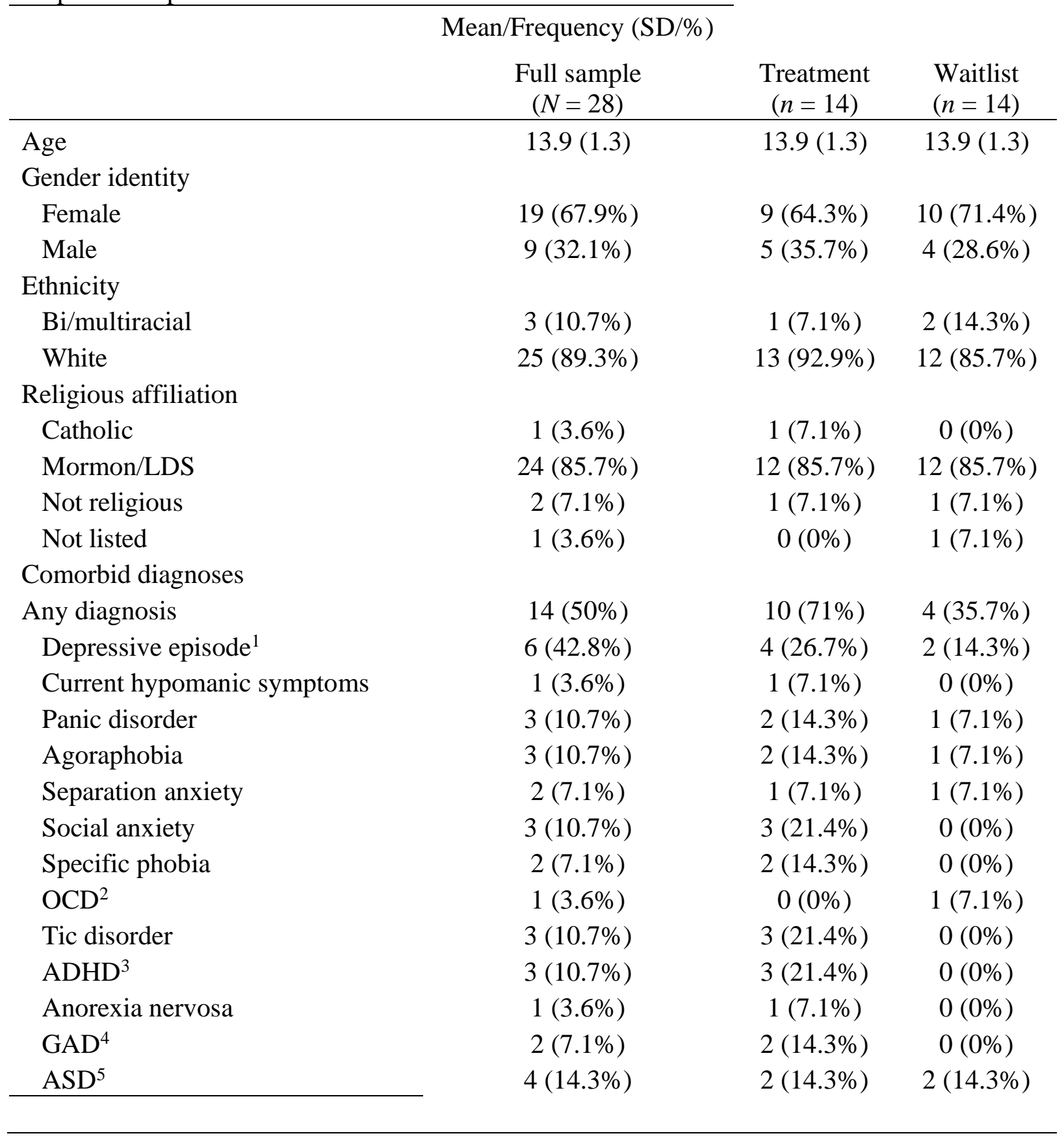

Note. LDS $=$ The Church of Jesus Christ of Latter-day Saints.

${ }^{1}$ Inclusive of past, present, and recurrent diagnoses, ${ }^{2}$ Obsessive compulsive disorder, ${ }^{3}$ Attention deficit hyperactivity disorder,

${ }^{4}$ Generalized anxiety disorder, ${ }^{5}$ Autism spectrum disorder cannot be ruled out 
Table 2

Regression Coefficients and 95\% Confidence Intervals From Best-Fitting Multilevel Models

\begin{tabular}{|c|c|c|c|c|c|c|c|}
\hline & $\begin{array}{l}\text { TSC-Y } \\
\text { Severity }\end{array}$ & $\begin{array}{l}\text { TSC-P } \\
\text { Severity }\end{array}$ & $\begin{array}{l}\text { TSC-Y } \\
\text { Distress }\end{array}$ & $\begin{array}{l}\text { TSC-P } \\
\text { Distress }\end{array}$ & YOQ-Y & YOQ-P & AAQ-TTM ${ }^{2}$ \\
\hline Intercept & $\begin{array}{l}1.44 \\
{[1.18 ; 1.71]^{*}}\end{array}$ & $\begin{array}{l}1.38 \\
{[1.21 ; 1.55]^{*}}\end{array}$ & $\begin{array}{l}0.86 \\
{[0.68 ; 1.05]^{*}}\end{array}$ & $\begin{array}{l}0.92 \\
{[0.75 ; 1.09]^{*}}\end{array}$ & $\begin{array}{l}34.73 \\
{[28.13 ; 41.33]^{*}}\end{array}$ & $\begin{array}{l}31.68 \\
{[24.36 ; 39.00]^{*}}\end{array}$ & $\begin{array}{l}37.50 \\
{[31.30 ; 43.70]^{*}}\end{array}$ \\
\hline Condition $^{1}$ & $\begin{array}{l}-0.16 \\
{[-0.53 ; 0.22]}\end{array}$ & & & & $\begin{array}{l}-22.46 \\
{[-31.83 ;-} \\
13.09]^{*}\end{array}$ & $\begin{array}{l}-10.94 \\
{[-21.41 ;-0.48]^{*}}\end{array}$ & $\begin{array}{l}9.86 \\
{[1.09 ; 18.62]^{*}}\end{array}$ \\
\hline $\begin{array}{l}\text { Week } \times \\
\text { Condition }\end{array}$ & $\begin{array}{l}0.06 \\
{[0.02 ; 0.09]^{*}}\end{array}$ & & & & & & $\begin{array}{l}-0.70 \\
{[-1.62 ; 0.22]}\end{array}$ \\
\hline $\mathrm{BIC}$ & 105.31 & 84.66 & 90.16 & 87.47 & 413.62 & 418.27 & 404.72 \\
\hline $\begin{array}{l}\text { Number of } \\
\text { observations }\end{array}$ & 51 & 50 & 51 & 50 & 51 & 50 & 51 \\
\hline $\begin{array}{l}\text { Number of } \\
\text { participants }\end{array}$ & 28 & 28 & 28 & 28 & 28 & 28 & 28 \\
\hline
\end{tabular}

* 0 outside the confidence interval.

${ }^{1}$ Relative to waitlist condition.

${ }^{2}$ Best-fitting model at $p<.10$.

Note. TSC-Y = Trichotillomania Scale for Children (Youth-Report); TSC-P = Trichotillomania Scale for Children (Parent-Report); YOQ-Y = Youth Outcomes Questionnaire (Youth-Report); YOQ-P = Youth Outcomes Questionnaire (Parent-Report); AAQ-TTM = Acceptance and Action Questionnaire-Trichotillomania; BIC = Bayesian Information Criterion. 
Table 3

Means, Standard Deviations, and Effect Sizes

\begin{tabular}{|c|c|c|c|c|c|c|}
\hline & \multicolumn{2}{|c|}{ Waitlist } & \multicolumn{2}{|c|}{ Treatment } & \multicolumn{2}{|c|}{ Hedges' $g$} \\
\hline & $\begin{array}{c}\text { Pre } \\
(\mathrm{n}=14)\end{array}$ & $\begin{array}{c}\text { Post } \\
(\mathrm{n}=14)\end{array}$ & $\begin{array}{c}\text { Pre } \\
(\mathrm{n}=14)\end{array}$ & $\begin{array}{c}\text { Post } \\
(\mathrm{n}=14)\end{array}$ & $\begin{array}{l}\text { Within- } \\
\text { group }^{1}\end{array}$ & $\begin{array}{l}\text { Between- } \\
\text { groups }\end{array}$ \\
\hline \multicolumn{7}{|l|}{ TSC Severity $^{2}$} \\
\hline Youth-report & $1.3(0.5)$ & $1.1(0.6)$ & $1.4(0.3)$ & $0.7(0.6)$ & -1.27 & -0.76 \\
\hline Parent-report & $1.4(0.5)$ & $1.2(0.3)$ & $1.4(0.4)$ & $1.0(0.5)$ & -0.70 & -0.71 \\
\hline \multicolumn{7}{|l|}{ TSC Distress $^{2}$} \\
\hline Youth-report & $0.8(0.6)$ & $0.6(0.4)$ & $0.9(0.5)$ & $0.5(0.5)$ & -0.77 & -0.26 \\
\hline Parent-report & $0.8(0.5)$ & $0.8(0.3)$ & $1.0(0.5)$ & $0.6(0.5)$ & -0.84 & -0.57 \\
\hline \multicolumn{7}{|l|}{$\mathrm{YOQ}^{2}$} \\
\hline Youth-report & $12.7(6.8)$ & $10.8(8.6)$ & $38.2(22.2)$ & $30.0(12.4)$ & -0.39 & 1.53 \\
\hline Parent-report & $20.1(12.8)$ & $22.5(14.7)$ & $35.5(20.0)$ & $27.7(15.4)$ & -0.39 & 0.26 \\
\hline AAQ-TTM $^{3}$ & $47.4(12.1)$ & $48.3(6.8)$ & $37.5(11.9)$ & 44.6 (13.9) & 0.51 & -0.21 \\
\hline
\end{tabular}

${ }^{1}$ Pre to posttreatment change in the treatment group.

${ }^{2}$ Higher scores reflect greater severity.

${ }^{3}$ Higher scores reflect more psychological flexibility.

Note . TSC = Trichotillomania Scale for Children; YOQ = Youth Outcomes Questionnaire; AAQ-TTM = Acceptance and Action Questionnaire-Trichotillomania. 

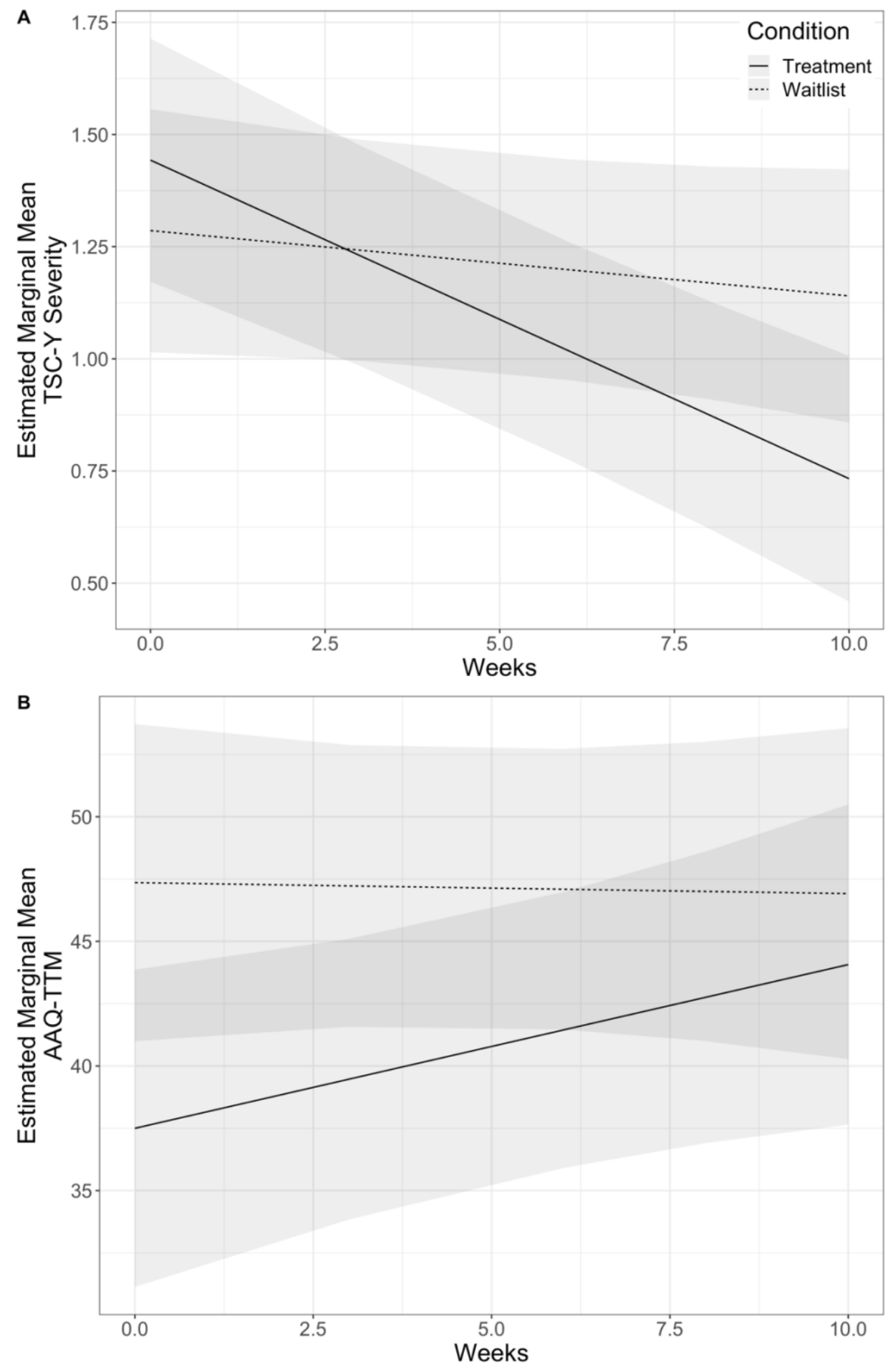

Figure 2. Estimated marginal means and standard error ribbons from best-fitting models for A) youth-reported TSC Severity at $p<.05$ and B) the AAQ-TTM at $p<.10$ (higher scores indicate improvement). These models included a week $\times$ condition interaction term. TSC $=$ Trichotillomania Scale for Children; AAQ-TTM = Acceptance and Action QuestionnaireTrichotillomania. 
AEBT for Adolescent Trichotillomania 41 